\title{
Control of Singlet Oxygen-induced Oxidative Damage in Escherichia coli
}

\author{
Sun Yee Kim, Eun Ju Kim and Jeen-Woo Park* \\ Department of Biochemistry, College of Natural Sciences, Kyungpook National University, Taegu 702-701, Korea
}

Received 28 January 2002, Accepted 22 March 2002

\begin{abstract}
Singlet oxygen $\left({ }^{1} \mathrm{O}_{2}\right)$ is a highly reactive form of molecular oxygen that may harm living systems by oxidizing critical cellular macromolecules. The oxy $R$ gene product regulates the expression of the enzymes and proteins that are needed for cellular protection against oxidative stress. In this study, the role of oxyR in cellular defense against a singlet oxygen was investigated using Escherichia coli oxyR mutant strains. Upon exposure to methylene blue and visible light, which generates singlet oxygen, the oxyR overexpression mutant was much more resistant to singlet oxygen-mediated cellular damage when compared to the oxy $R$ deletion mutant in regard to growth kinetics, viability and protein oxidation. Induction and inactivation of major antioxidant enzymes, such as superoxide dismutase and catalase, were observed after their exposure to a singlet oxygen generating system in both oxy $R$ strains. However, the $\operatorname{oxy} R$ overexpression mutant maintained significantly higher activities of antioxidant enzymes than did the oxyR deletion mutant. These results suggest that the oxyR regulon plays an important protective role in singlet oxygen-mediated cellular damage, presumably through the protection of antioxidant enzymes.
\end{abstract}

Keywords: Singlet oxygen, oxyR regulon, Protein damage, Catalase, Superoxide dismutase

\section{Introduction}

Singlet molecular oxygen $\left({ }^{1} \mathrm{O}_{2}\right)$, an electronically-excited state of oxygen that is the result of the promotion of an electron to high-energy orbitals, is produced in mammalian cells under normal and pathophysiological conditions (Kanofsky, 1989). The photodynamic action of some drugs and pigments is also mediated through ${ }^{1} \mathrm{O}_{2}$ (Weishaupt et al., 1976). ${ }^{1} \mathrm{O}_{2}$ is a highly reactive form of molecular oxygen that may harm living systems by oxidizing critical cellular macromolecules, including lipids, nucleic acids and proteins. It also promotes

*To whom correspondence should be addressed.

Tel: 82-53-950-6352; Fax: 82-53-943-2762

E-mail: parkjw@knu.ac.kr deleterious processes, such as lipid peroxidation, membrane damage and cell death (Halliwell and Gutteridge, 1999).

The presence of a global regulatory mechanism for the coordinate expression of enzymes and proteins that are needed for cellular protection against oxidative stress is well documented (Christman et al., 1985; Morgan et al., 1986; Storz et al., 1987). The induction of the oxidative response regulon is under positive control by the oxyR gene product, whose expression or activity is oxidant-inducible (Christman et al., 1985). The Escherichia coli mutant oxyR2 carries a dominant mutation that confers resistance to a variety of oxidizing agents. It also causes the overexpression of several enzymes that are involved in defenses against oxidative damage, whereas the deletions of $o x y R$ are recessive and highly sensitive to oxidant (Christman et al., 1985).

In the present study, the role of oxyR in cellular defense against a singlet oxygen-producing system was investigated using the $\operatorname{oxy} R$ overexpression mutant strain TA4110 and the $\operatorname{oxy} R$ deletion mutant strain TA4112. The two strains were expected to exhibit differences in regard to sensitivity to the toxic effects of singlet oxygen. To determine if such differences do indeed exist between the two types of cells, we examined growth kinetics, viability, modulation of activities of major antioxidant enzymes, and oxidative damage to protein upon exposure to methylene blue and visible light (MB/light), which generates singlet oxygen (Tabatabaie and Floyd, 1994). The data suggest that the $o x y R$ regulon plays an important protective role in singlet oxygen-mediated cellular damage, presumably through the protection of antioxidant enzymes.

\section{Materials and methods}

Materials $\mathrm{MB}$, 2,4-dinitrophenylhydrazine (DNPH), pyrogallol, and hydrogen peroxide were obtained from Sigma (St. Louis, USA). A Bio-Rad protein assay kit was obtained from Bio-Rad (Hercules, USA).

Bacterial strains and culture conditions The $E$. coli strains that harbored the oxyR2 (TA4110, oxyR-constitutive) and the oxy $\Delta 3$ (TA4112, oxyR deletion) genes were a kind gift of Dr. B. N. Ames (University of California, Berkeley). A minimal salt medium that 
was supplemented with $0.5 \%$ glucose, $0.05 \%$ yeast extract and $10 \mathrm{mg} / \mathrm{ml}$ each arginine and methionine was used. It was designated as a GY medium. The rich medium, TSY, contained 3\% trypticase soy broth plus $0.5 \%$ yeast extract. Overnight cultures that were grown in the $\mathrm{GY}$ medium at $37^{\circ} \mathrm{C}$ on a rotary shaker at $200 \mathrm{rpm}$ were used to inoculate the fresh GY and TSY media. Exposure to $\mathrm{MB} /$ light began when the optical density $(600 \mathrm{~nm})$ reached 0.3-0.4. Bacterial cultures with various concentrations of MB were irradiated with white light from a $100 \mathrm{~W}$ tungsten lamp. Aliquots of the exposed cells were removed at time intervals and assayed for changes in absorbance at $600 \mathrm{~nm}$. Viability was determined at various times by removing the samples and performing viable cell counts.

Protein carbonyl content The protein carbonyl content was determined spectrophotometrically using the described DNPHlabeling procedure (Levine et al., 1994). The crude extract ( 1 mg protein) was incubated with $0.4 \mathrm{ml} 0.2 \% \mathrm{DNPH}$ in $2 \mathrm{M} \mathrm{HCl}$ for $1 \mathrm{~h}$ at $37^{\circ} \mathrm{C}$. The protein hydrazone derivatives were sequentially extracted with $10 \%(\mathrm{w} / \mathrm{v})$ trichloroacetic acid, treated with ethanol/ ethyl acetate, $1: 1(\mathrm{v} / \mathrm{v})$, and re-extracted with $10 \%$ trichloroacetic acid. The resulting precipitate was dissolved in $6 \mathrm{M}$ guanidine hydrochloride. The sample's spectrum difference, which was treated with $\mathrm{DNPH}$ in $\mathrm{HCl}$, was examined versus a sample that was treated with $\mathrm{HCl}$ alone. Results are expressed as nmole of DNPH incorporated per $\mathrm{mg}$ of protein that was calculated from an absorbtivity of $21.0 \mathrm{mM}^{-1} \mathrm{~cm}^{-1}$ at $360 \mathrm{~nm}$ for aliphatic hydrazones.

Activity of antioxidant enzymes The bacterial cells were collected at $10,000 \times g$ for $10 \mathrm{~min}$ at $4^{\circ} \mathrm{C}$ and washed once with cold $50 \mathrm{mM}$ potassium phosphate/0.1 mM EDTA at $\mathrm{pH} 7.8$ before being resuspended in $1.0 \mathrm{ml}$ of this buffer. Cell-free extracts were prepared by sonication. Cellular debris was removed by a $10-\mathrm{min}$ centrifugation at $15,000 \times g$ at $4^{\circ} \mathrm{C}$. The supernatant was collected, and the protein levels were determined by the method of Bradford using bovine serum albumin as the standard. Catalase activity was measured in terms of the decomposition of hydrogen peroxide, which was followed directly by a decrease in absorbance at $240 \mathrm{~nm}$ (Beers and Sizer, 1952). SOD activity in the cell extracts was assayed spectrophotometrically using the pyrogallol assay as described (Kim et al., 2001). One unit of activity is defined as the quantity of enzyme that reduces the superoxide-dependent color change by $50 \%$.

In vitro inactivation Cell-free extracts of normally grown $E$. coli cells were exposed to $25 \mu \mathrm{M} \mathrm{MB}$ plus light and assayed for activities of SOD and catalase as described previously.

Statistical analysis A statistical analysis was performed by the student's $t$-test. A $p$-value of less than 0.05 was considered statistically significant.

\section{Results}

The growth kinetics of the TA4110 and TA4112 strains were compared following exposure to a singlet oxygen-generating system. Under aerobic conditions, the growth of both oxyR
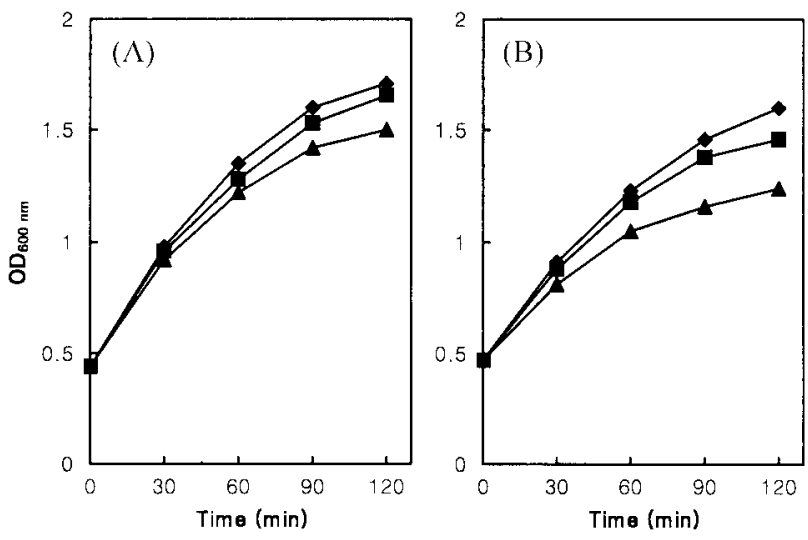

Fig. 1. Growth of TA4110 (A) and TA4112 (B) upon exposure to $\mathrm{MB} /$ light. Exponentially growing bacteria were cultured at $37^{\circ} \mathrm{C}$ with shaking and exposed to $\mathrm{MB}$ and visible light. Increasing the optical densities of the cultures monitored cell growth. The result is representative of the three separate experiments. $\downarrow$, No addition; $\boldsymbol{\square}, 10 \mu \mathrm{M} \mathrm{MB} ; \boldsymbol{\Delta}, 25 \mu \mathrm{M} \mathrm{MB}$.

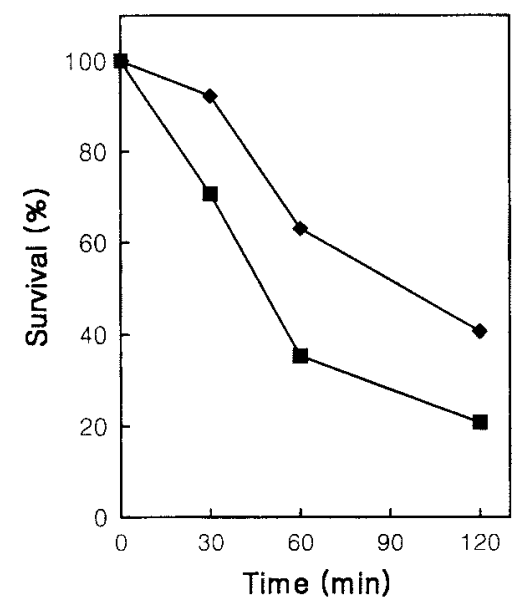

Fig. 2. Effect of $\mathrm{MB} /$ light on viability of $\operatorname{oxyR}$ cells. After the cells were exposed to $25 \mu \mathrm{M} \mathrm{MB}$ and various times of illumination, the viability was determined, as described in the text. The result is representative of the three separate experiments. $\bullet$, TA4110; $\boldsymbol{\square}, \mathrm{TA} 4112$.

strains were inhibited by $\mathrm{MB} /$ light in a concentrationdependent manner; however, the growth inhibition was more pronounced in TA4112 than in TA4110 (Fig. 1). Growth inhibition was also accompanied by the loss of cell viability. To determine the rate of viability loss, early exponential phase cells were incubated in liquid culture in the presence of $\mathrm{MB}$ with illumination, and subsequently plated to determine the colony-forming ability. The exponential phase was chosen since cells can become resistant to various forms of stress when they enter the stationary phase. The TA4110 cells were much more resistant to exposure of MB/light than TA4112 cells (Fig. 2). The TA4110 cells showed a 63\% survival rate after exposure to $25 \mu \mathrm{M} \mathrm{MB}$ and illumination for $60 \mathrm{~min}$ compared with the TA4112 mutant cells, which showed a 


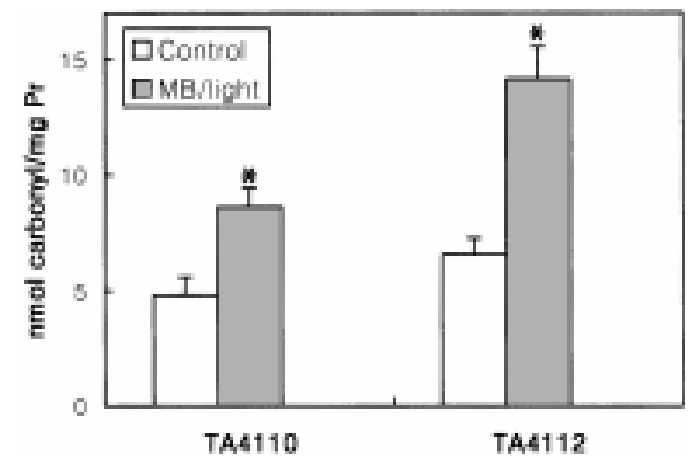

Fig. 3. Protein carbonyl content of $\operatorname{oxy} R$ strains exposed to $25 \mu \mathrm{M}$ MB plus $2 \mathrm{~h}$ illumination. Protein carbonyls were measured in the cell-free extracts by the method of Levine and co-workers with the use of 2,4-dinitrophenylhydrazine (Levine et al., 1994). Results are the mean \pm S.D. of the three determinations. ${ }^{*} p<0.01$ compared to untreated cells.

$32 \%$ survival rate. Exposure of oxyR strains to $\mathrm{MB}$ in the absence of illumination, or by illumination alone, did not modify the viability of cells. The strain-deleted $\operatorname{oxy} R$ was killed to a greater extent upon exposure to MB/light, indicating that $\operatorname{oxy} R$ may be involved in protecting the cells from singlet oxygen-mediated cellular damage.

Protein oxidation that is mediated by the reactive oxygen species is accompanied by the conversion of proline, lysine, arginine and histidine residues into carbonyl derivatives (Amici et al., 1989), therefore providing a convenient assay of oxidative modification. The carbonyl content of the protein can be measured using a phenylhydrazine formation reaction. We measured the carbonyl levels for protein oxidation $2 \mathrm{~h}$ after cellular exposure to $25 \mu \mathrm{M} \mathrm{MB} /$ light in order to determine whether the $\operatorname{oxy} R$ expression decreased sensitivity to protein damage. As shown in Fig. 3, protein oxidation was significantly increased in both strains of the incubation with MB/light. However, the TA4112 cells exhibited significantly higher levels of carbonyl groups compared to TA4110.

To determine whether the expression of the $\operatorname{oxy} R$ gene induces concomitant alteration in the activity of major antioxidant enzymes, the modulation of the activities of catalase and SOD upon exposure to MB/light was examined. When the two $\operatorname{oxy} R$ strains were exposed to MB/light, the catalase activity increased; however, significantly higher activity was observed in TA4110 compared to TA4112 (Fig. 4). The activity of SOD similarly increased in the two oxyR strains upon exposure to $\mathrm{MB} /$ light, although slightly lower activity was observed in TA4112. These data do not, however, establish whether this increase was due to de novo synthesis or to the activation of pre-existing proteins. The effect of MB/ light on the activities of antioxidant enzymes was examined in cell-free extracts that lacked induction capabilities. Fig. 5 demonstrates that antioxidant enzymes in the two oxyR strains were inactivated upon exposure to $\mathrm{MB} /$ light in a timedependent manner, although the rate of inactivation was slower in TA 4110 cells. These results indicate that
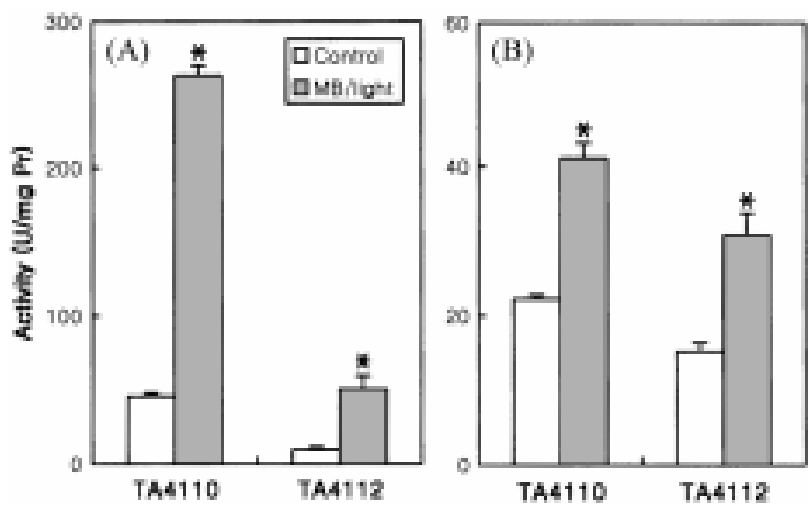

Fig. 4. Effect of $\mathrm{MB}(25 \mu \mathrm{M}) /$ light $(2 \mathrm{~h})$ on the activities of catalase (A) and SOD (B) in oxyR strains. Results are the mean \pm S.D. of the three determinations. $* p<0.01$ compared to untreated cells.
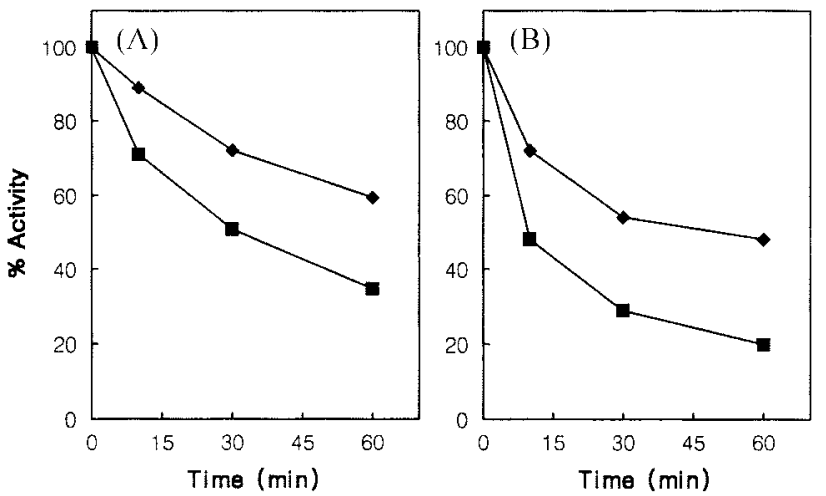

Fig. 5. Inactivation of catalase (A) and SOD (B) in cell-free extracts from oxyR strains upon exposure to $25 \mu \mathrm{M} \mathrm{MB}$ plus visible light. Activity of untreated cell-free extract is expressed as $100 \%$. Results represent the means of the three determinations. TA4110; $\mathbf{\square}$, TA4112.

antioxidant enzymes are susceptible to the damage that is caused by MB/light. This observation suggests that singlet oxygen, besides inducing the synthesis of catalase and superoxide dismutase, could also cause inactivation of these enzymes.

\section{Discussion}

We know that ${ }^{1} \mathrm{O}_{2}$ can be generated in cells, such as under conditions of oxidative stress (Sies, 1986), decomposition of lipid peroxides, or by spontaneous dismutation of superoxide (Krinsky, 1974; Foote, 1977). In addition, both naturally occurring compounds such as riboflavin (Joshi, 1985) and many xenobiotics, such as psoralene (de Mol et al., 1981), porphyrins (Weishaupt et al., 1976) and tetracyclins (Hasan and Khan, 1986), can generate ${ }^{1} \mathrm{O}_{2}$ inside cells when irradiated by visible light. Singlet oxygen is usually generated in vitro by photosensitization reactions. Irradiation of the sensitizer dye, such as $\mathrm{MB}$ or $\mathrm{RB}$, mediates photoreactions through an 
excited triplet state. It acts either by hydrogen atom or electron transfer reactions (type I) or by transferring the excited energy, forming singlet oxygen, which then reacts with the target molecules (type II) (Foote, 1977).

$$
\begin{aligned}
& \mathrm{MB}+\text { light } \rightarrow \mathrm{MB}^{* 1} \text { (singlet) } \rightarrow \mathrm{MB}^{* 3} \text { (triplet) } \\
& \mathrm{MB}^{* 3} \rightarrow \mathrm{H} \text { or e transfer (type I) } \\
& \mathrm{MB}^{* 3}+\mathrm{O}_{2} \rightarrow{ }^{1} \mathrm{O}_{2}+\mathrm{MB} \text { (type II) }
\end{aligned}
$$

Light induced a few diseases, including erythropoietic protoporphyria, pellagra and cataractogenesis that have been attributed in part to the toxicity of ${ }^{1} \mathrm{O}_{2}$ (Mathews-Roth, 1986; Egorov et al., 1987). On the other hand, singlet oxygen is the primary active species in a novel cancer treatment modality known as photodynamic therapy (Henderson and Dougherty, 1992).

This study demonstrated that the oxidative defense regulon $o x y R$ plays an important role in protection from toxicity that is induced by singlet oxygen. We have shown that the oxyR deletion mutant is hypersensitive to damage that is caused by singlet oxygen. Cells that lack the expression of the oxyR gene show a significant decrease in cell viability (Fig. 2) and increase in oxidative damage to the protein (Fig. 3). This finding emphasizes the essential role of the oxyR regulon in mitigating such damage.

Although antioxidant enzymes play a major role in the cellular defense mechanism, they are susceptible to inactivation by reactive oxygen species. The oxidative processes result in the loss of key antioxidant enzymes (Kono and Fridovich, 1982; Tabatabaie and Floyd, 1994), which may exacerbate oxidative stress-mediated cytotoxicity. However, it is also possible that prokaryotes and eukaryotes compensate for the inactivation of antioxidant enzymes by the enhanced expression of SOD, catalase, and other antioxidant enzymes. Induction of antioxidant proteins in response to oxidative stress in E. coli and Salmonella typhimurium, as well as mammalian cells, is well known (Christman et al., 1985; Greenberg et al., 1990). Our results show that catalase and SOD activities were induced, and that the activity of catalase in the $\operatorname{oxy} R$ overexpression mutant is significantly higher than that in the oxyR deletion mutant. In contrast, the activity of SOD in TA4110 was higher to a lesser extent in the absence or presence of MB/light compared to TA4112 (Fig. 4). The SOD activity is not regulated by $\operatorname{xxy} R$ (Demple and Halbrook, 1983; Bowen and Hassan, 1988), although the oxyRoverexpressed mutant constitutively produces a high level of catalase, and presumably other antioxidant proteins. The soxR regulon positively controls $\sim 15$ genes in E. coli including SOD (Greenberg et al., 1990). Singlet oxygen may play a role directly or indirectly in the soxR regulon-mediated induction of SOD. When cell-free extracts from both $\operatorname{oxy} R$ strains were exposed to singlet oxygen, the catalase and SOD in TA4112 were more susceptible to oxidative inactivation (Fig. 5). These in vivo and in vitro results indicate that a higher level of some antioxidant enzymes may protect catalase and SOD from damage that is mediated by singlet oxygen. It may eventually prevent cells from progressing to the pro-oxidant state. This protection may be a mechanism that increases survival and decreases protein damage in the $o x y R$ overexpression mutant.

In conclusion, results of this study indicate that a deficiency of $o x y R$ increased the sensitivity of $E$. coli to the singlet oxygen-generating system, such as $\mathrm{MB} /$ light. The oxyR regulon also provides protection against the oxidative inactivation of SOD and catalase through the induction of antioxidant enzymes. These results provide support for the role of oxyR as an important regulator to protect cells against singlet oxygen-mediated oxidative stress.

Acknowledgment This work was supported by a grant (HMP-00-B-20800-0071) from the Ministry of Health and Welfare, Korea.

\section{References}

Amici, A., Levine, R. L., Tsai, L. and Stadtman, E. R. (1989) Conversion of amino acid residues in proteins and amino acid homopolymers to carbonyl derivatives by metal-catalyzed oxidation reactions. J. Biol. Chem. 264, 3341-3346.

Beers, R. F. Jr and Sizer, I. W. (1952) A spectrophotometric method for measuring the breakdown of hydrogen peroxide by catalase. J. Biol. Chem. 195, 133-140.

Bowen, S. W. and Hassan, H. M. (1988) Induction of the manganese-containing superoxide dismutase in Escherichia coli is independent of the oxidative stress (oxyR-controlled) regulon. J. Biol. Chem. 263, 14808-14811.

Christman, M. F., Morgan, R. W., Jacobson, F. S. and Ames, B. N. (1985) Positive control of a regulon for defenses against oxidative stress and some heat-shock proteins in Salmonella typhimurium. Cell 41, 753-762.

de Mol, N. J., van Henegouven, G. M. J. B. and van Beele, B. (1981) Singlet oxygen formation by sensitization of furocoumarins complexed with, or bound covalently to DNA. Photochem. Photobiol. 34, 661-666.

Demple, B. and Halbrook, J. (1983) Inducible repair of oxidative DNA damage in Escherichia coli. Nature 304, 466-468.

Egorov, S. I., Babizhaev, M. A., Krasnovskii, A. A. Jr and Shredova, A. A. (1987) Photosensitized generation of singlet molecular oxygen by endogenous substances of the eye lens. Biofizika 32, 169-171.

Foote, C. S. (1977) Singlet oxygen; in Free Radicals in Biology, Pyror, W. A. (ed.), pp. 85-133, Vol. 2, Academic Press, New York, New York.

Greenberg, J. T., Monach, P., Chou, J. H., Josephy, P. D. and Demple, B. (1990) Positive control of a global antioxidant defense regulon activated by superoxide-generating agents in Escherichia coli. Proc. Natl. Acad. Sci. USA 87, 6181-6185.

Halliwell, B. and Gutteridge, J. M. C. (1999) Free Radicals in Biology and Medicine, Oxford Press, Oxford, UK.

Hasan, T. and Khan, A. U. (1986) Phototoxicity of the tetracyclines: photosensitized emission of singlet delta dioxygen. Proc. Natl. Acad. Sci. USA 83, 4604-4606.

Henderson, B. W. and Dougherty, T. J. (1992) How does photodynamic therapy work? Photochem. Photobiol. 55, 145157. 
Joshi, P. C. (1985) Comparison of the DNA-damaging property of photosensitized riboflavin via singlet oxygen $\left({ }^{1} \mathrm{O}_{2}\right)$ and superoxide radical $\mathrm{O}_{2}^{-}$mechanisms. Toxicol. Lett. 26, 211-217.

Kanofsky, J. R. (1989) Singlet oxygen production by biological systems. Chem. Biol. Interact. 70, 1-28.

Kim, S. Y., Kim, R. H., Huh, T. L. and Park, J.-W. (2001) $\alpha-$ Phenyl-N-t-butylnitrone protects oxidative damage to HepG2 cells. J. Biochem. Mol. Biol. 34, 43-46.

Kono, Y. and Fridovich, I. (1982) Superoxide radical inhibits catalase. J. Biol. Chem. 257, 5751-5754.

Krinsky, N. I. (1974) Membrane photochemistry and photobiology. Photochem. Photobiol. 20, 532-535.

Levine, R. L., Williams, J. A., Stadtman, E. R. and Shacter, E. (1994) Carbonyl assays for determination of oxidatively modified proteins. Methods Enzymol. 233, 346-357.

Mathews-Roth, M. M. (1986) Beta-carotene therapy for erythropoietic protoporphyria and other photosensitivity diseases. Biochimie 68, 875-884.
Morgan, R. W., Christman, M. F., Jacobson, F. S., Storz, G. and Ames, B. N. (1986) Hydrogen peroxide-inducible proteins in Salmonella typhimurium overlap with heat shock and other stress proteins. Proc. Natl. Acad. Sci. USA 83, 8059-8063.

Sies, H. (1986) Biochemistry of oxidative stress. Angew. Chem. Int. Ed. Engl. 25, 1058-1072.

Storz, G., Christman, M. F., Sies, H. and Ames, B. N. (1987) Spontaneous mutagenesis and oxidative damage to DNA in Salmonella typhimurium. Proc. Natl. Acad. Sci. USA 84, 89178921.

Tabatabaie, T. and Floyd, R. A. (1994) Susceptibility of glutathione peroxidase and glutathione reductase to oxidative damage and the protective effect of spin trapping agents. Arch. Biochem. Biophys. 314, 112-119.

Weishaupt, K. R., Gomer, C. J. and Dougherty, T. J. (1976) Identification of singlet oxygen as the cytotoxic agent in photoinactivation of a murine tumor. Cancer Res. 36, 23262329. 